\title{
A Linear Discriminator of Width
}

\author{
José A.F. Leite ${ }^{\star}$ and Edwin R. Hancock \\ University of York,Computer Science Department \\ YO1 5DD, York,England
}

\begin{abstract}
Our aim in this paper is to present a methodology for linearly combining multi-channel filter information, using principal components analysis. We take as our application vehicle the detection and classification according to width of line-features. Here we operate on the raw data with oriented multi-scale line detection kernels. The objective in this study is to develop a linear discriminator of line width which can be used to classify the detected features. By performing principal components analysis on the response of the multi-scale filter bank to a set of example line-profiles, we transform the filter-outputs. Under this transformation, the principal component of maximum variance is demonstrated to have linear dependance upon line-width. We demonstrate the utility of our line-classification process under conditions of controlled noise.
\end{abstract}

\section{Introduction}

Over the past decade, the rapidly emergent paradigm of multichannel analysis has proved to be an overwhelmingly powerful technique for extracting information concerning the properties of intensity features $[1,5,6,7]$. Basic to the approach is the idea that intensity structure can be represented by the output of a filter bank that has channels specialized to the selection of different orientation $[1]$, scale [5] or symmetry [6, 7]. Examples include the use of oriented quadrature filter pairs to detect lines or edges $[1,7]$ and the use of Gaussian wavelets for multiscale edge detection [5].

Whatever the nature of the filter-bank being used to characterise the different types of intensity structure, a framework is required for combining the individual channel responses and making decisions concerning the identity of the underlying image features. In the quadrature filtering approach [6] this is done by adding the squares of the even and odd symmetry filter responses to construct a local energy measure and using their relative phase [1] to separately label edge and line artifacts. In the wavelet approach the multiscale persistence of features can be modelled by the use of Lipschitz exponents [5] or by coupled membranes [4]. Adopting a statistical standpoint, Leite and Hancock [7] have shown how the Mahalanobis distance can be used as a fusional device for integrating together the information represented by a multiscale filter-bank for subsequent processing by

\footnotetext{
* This work is being sponsored in part by the Brazilian Research Council(CNPq)
} 
non-linear relaxation operations. Taking these ideas one stage further, Hancock [2] has demonstrated that by adopting a phase representation of sine and cosinephase S-Gabor filter responses, edges and lines can be simultaneously detected and discriminated using relaxation operations.

Central to each of the channel combination models described above is the requirement for highly non-linear processing operations. Although these existing methods are certainly effective, they may be regarded as somewhat premature in their rejection of simpler linear processing operations.

Our aim in this paper is to describe how the channel responses can be combined in a linear way using principal component analysis [8] to discriminate between different classes of intensity features. Although we have selected the task of discriminating line-features of differing width to demonstrate the utility of our ideas, the framework is potentially applicable to a multitude of feature interpretation problems. In essence, principal components analysis allows the multichannel feature-vector to be linearly transformed so that it is alligned with the direction of maximum variance for a set of training examples. Components of insignificant variance may then be discarded. In our line-discrimination example, the training patterns are provided by idealised examples of known width, while the filter bank consists of second-derivative of Gaussian operators of varying scale. Here the principal component of maximum variance is shown to be linearly dependant on line-width. Moreover, the resulting width discrimination process is shown to be robust to severe levels of noise.

The outline of this paper is as follows. In Sect. 2 we describe the ingredients of our multi-scale filtering approach. Section 3 describes the statistical framework and principal components analysis. Experiments are presented in Sect. 4 and conclusions are offered in Sect. 5 .

\section{Multichannel Filtering}

We are interested in identifying a filter basis consisting of oriented even symmetry kernels that can be used to characterise directional line features at a variety of different spatial scales. There are several alternatives available to us including directional second derivatives of the Gaussian and the cosine phase S-Gabor functions [2]. However, since our main concern here is proof of concept, we will not be interested in optimal choice of filter basis and we therefore choose to work with the second-derivative of Gaussian due to its parametric simplicity. The kernel of scale $s$ and orientation $\theta$ is as follows

$$
L_{\theta, s}(x, y)=\left(1-\frac{(x \cos \theta+y \sin \theta)^{2}}{s^{2}}\right) \exp \left[-\frac{x^{2}+y^{2}}{2 s^{2}}\right]
$$

It is readily verifiable that this filter is of zero d.c. bias, i.e. that the result of the double integration $\int_{-\infty}^{\infty} \int_{-\infty}^{\infty} L_{\theta, s}(x, y) d x . d y$ is equal to zero.

The channel responses are obtained in the usual way, by convolving multiscale filter kernels with the available image data $I(x, y)$

$$
R_{\theta, s}(x, y)=\int_{-\infty}^{\infty} \int_{-\infty}^{\infty} L_{\theta, s}(u, v) I(x-u, y-v) d u . d v
$$


We are interested in constructing feature-vectors from the output of oriented multiscale filters which form a dyadic basis. This is in line with the physiology of natural vision systems, which are known to employ three different scales each separated by one octave. At each of the orientation states of the filter basis the filter responses of different scale are stacked to form the following feature vector for the lines in the image data

$$
\mathbf{F}_{\theta}(x, y)=\left(\begin{array}{c}
R_{\theta, s}(x, y) \\
R_{\theta, 2 s}(x, y) \\
R_{\theta, 4 s}(x, y)
\end{array}\right)
$$

In the work reported here we use two orientation states aligned along the vertical and horizontal axes of the pixel lattice. The filter stacks of different orientation can be amalgamated to give the combined multiscale feature-vector $\mathbf{W}(x, y)^{T}=$ $\left(\mathbf{F}_{0}(x, y) \quad \mathbf{F}_{\frac{\pi}{2}}(x, y)\right)^{T}$.

\section{Principal Components Analysis}

In order to establish a set of training examples for principal components analysis, we construct a series of idealised line-profiles of known width $w$. For each such profile, we compute the set of responses for the distinct kernels of the filter bank and such responses are stacked to form a normalised pattern-vector $\mathbf{U}_{w}$.

Our basic aim in performing principal components analysis on the output of the multichannel filter bank, is to identify the linear transformation of the individual filter reponses that has maximum variance with respect to the set of example line-profiles [8]. Underpinning this analysis is the observation that if $F_{\theta, s}(x, y)$ is a random-vector with partially correlated components, then we can perform a linear transformation of the individual instances into a new coordinate space. The axes of this new feature-space are the eigenvectors of the covariance matrix for the example profiles. The associated eigenvalues are the fractions of total variance residing in the corresponding components of the transformed vectors. Since the different components of the transformed feature-vector are naturally graded according to their degree of intrinsic variation, they may be systematically examined to determine their dependance on line-width.

The basic computational ingredient of principal components analysis is the covariance matrix for the components of the feature-vector evaluated over the set of example profiles. If $\boldsymbol{\mu}=E\left[\mathbf{U}_{w}\right]$ is the mean feature vector, then the covariance matrix is equal to $\Sigma=E\left[\left(\mathbf{U}_{w}-\boldsymbol{\mu}\right)\left(\mathbf{U}_{w}-\boldsymbol{\mu}\right)^{T}\right]$.

The principal components transformation is obtained by solving the eigenvalue equation $|\Sigma-\lambda I|=0$, where $I$ is the $3 \times 3$ identity matrix. The eigenvalues $\lambda_{1}, \lambda_{2}$ and $\lambda_{3}$ are the variances of the components of the transformed feature-vectors for the example line profiles. Associated with each of these three eigenvalues is an eigenvector $\mathbf{V}_{\lambda}$ whose components satisfy the system of linear equations given by $\Sigma \mathbf{V}_{\lambda}=\lambda \mathbf{V}_{\lambda}$.

These eigenvectors are the axes of a new orthonormal co-ordinate system. Specifically, the three eigenvectors are the columns of the transformation matrix 
between the original feature-vectors and the principal components representation. If we denote the transformation matrix by $\Theta=\left(\mathbf{V}_{\lambda_{1}}, \mathbf{V}_{\lambda_{2}}, \mathbf{V}_{\lambda_{3}}\right)$, then the transformed feature-vectors are

$$
\left(\begin{array}{l}
C_{\theta, 1}(x, y) \\
C_{\theta, 2}(x, y) \\
C_{\theta, 3}(x, y)
\end{array}\right)=\Theta^{T} \cdot \mathbf{F}_{\theta}(x, y)-\mu
$$

Figure 1 shows the three principal components extracted from the example lineprofiles as a function of line-width. The striking feature of these plots is the near linear dependance of the first principal component upon line-width. Superimposed on the extracted values of the first principal component is the result of a simple linear fit. It is this linear interpolation of the principal component data that we use as our discriminator of line-width. The fraction of variance residing in this leading component is $96 \%$. By contrast, in the second principal component, the associated eigenvalue accounts for less than $4 \%$ of the total variance of the example line profiles. The fractional variance in the third component is negligible.

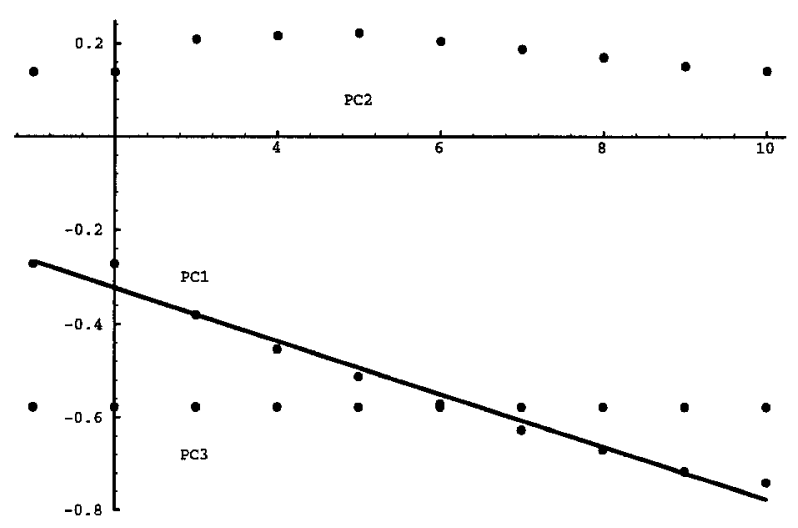

Fig. 1. First,second and third principal components

\section{Experiments}

In order to demonstrate the effectiveness of our width discrimination technique under conditions of controlled noise, we have generated a series of synthetic images. Each image consists of 7 concentric circles with widths varying from 2 to 8 pixels. The inner circle has a radius of 30 pixels and for the others, this values is increased by 20 pixels plus the width. The grey-level of the circles is 157 while that of the background is 57 . To each image we have added Gaussian 
noise of zero mean and fixed variance. Figure 2 shows the results of applying our technique to noise-free data and in Fig 3 the same results to the same image in which the signal-to-noise ratio (SNR) is 25:10. From top to bottom and from left to right, the subimages show the original picture, the combined line-map and the six width-maps for $w \in\{2,3,4,5,6,7\}$ pixels.

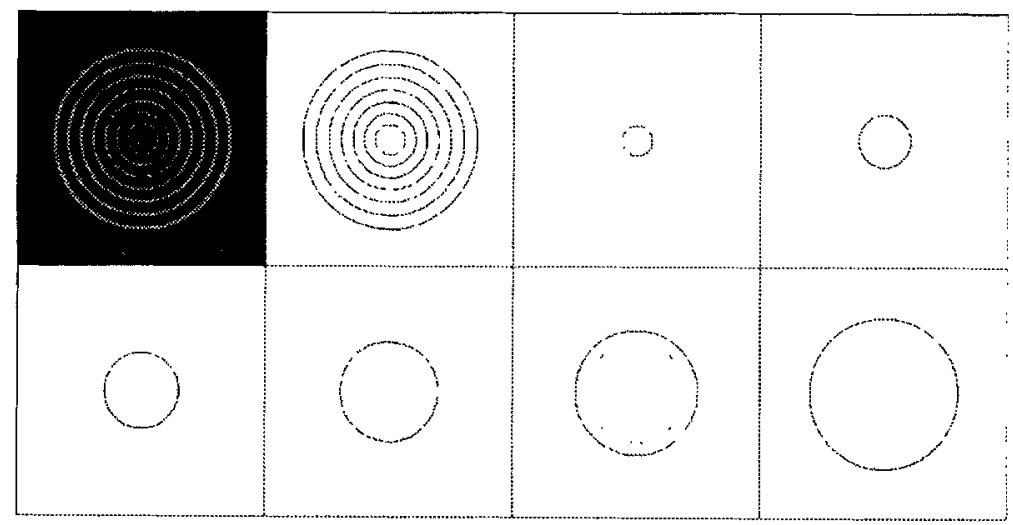

Fig. 2. Width discrimination on a noise-free image

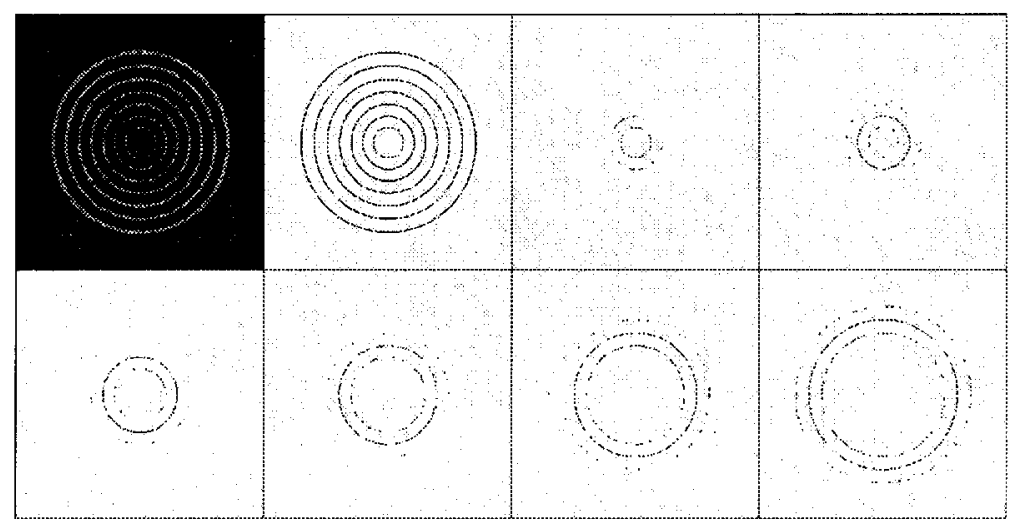

Fig. 3. Width discrimination on an SNR $=25: 10$ image

These results are very encouraging. Under conditions of zero noise the discrimination is almost perfect. At intermediate noise levels when $\mathrm{SNR}=25: 6$, some 
$75.85 \%$ of pixels are correctly classified according to width; the degree of contaminating error comes only from the two neighbouring width-bins. At the highest noise level when $\mathrm{SNR}=25: 10$, although the fraction of contamination has increased to $36.78 \%$, the bulk of the classification errors are still only a single width bin away from the ideal result.

We have chosen to use filters of three different scales (i.e. $s=1, s=2$ and $s=4$ ) on the basis of physiological evidence that suggests that the channels in the visual cortex are separated by approximately one octave. We have also repeated the experiments described above with both a larger number of filters and with a more dense coverage of the range of scale. Although the results are in some cases slightly superior, their use is not justifiable on the grounds of additional computational cost.

\section{Conclusions}

We have described a linear approach to texture discrimination. The technique relies on performing a principal components analysis of a series of example line profiles to identify linear combinations of filter response from a multiscale filter bank. We demonstrate that the principal component of maximum variance has an approximately linear dependance of line-width. When applied to the classification of variable-width line structure subjected to controlled levels of noise, the resulting discriminator is demonstrated to operate robustly provided that the SNR does not fall below 25:10.

\section{References}

1. E. Adelson and W.T. Freeman, "The design and use of steerable filters", IEEE PAMI, PAMI 13, pp. 891-906. 1991.

2. E. R. Hancock, "Resolving Edge-line Ambiguities by Relaxation Labelling", Proceedings of IEEE CVPR Conference, pp. 300-306, 1993.

3. T.S. Lee, D Mumford and A Yuille, "Texture Segmentation by Minimising VectorValued Functionals: The Coupled Membrane Model", Proceedings of the Second European Conference on Computer Vision, pp 165-173, 1992.

4. S. Mallat and S Zhong, "Characterisation of signals from multiscale edges", IEEE PAMI, PAMI 14, pp. 710-732. 1992.

5. M C Morrone and R Owens, "Feature detection using local energy", Pattern Recognition Letters, 6, pp 303-313, 1987.

6. P. Perona and J. Malik, "Detecting and localizing edges composed of steps, peaks and roofs", Proceedings of the Third Int. Conf. on Comp. Vision, pp. 52-57, 1990.

7. J.A.F.Leite and E.R.Hancock, "Statistically combining and refining multi-channel information", Progress in Image Analysis III: Editor S.Impedovo, pp. 193-200, 1993.

8. P. Devijver and J.Kittler, "Pattern Recognition: A Statistical Approach", PrenticeHall, 1982. 